\title{
Experimental study of paint coatings resistance to osmotic destruction depending on water-soluble salts content on a metal surface
}

\author{
Allanazar Atdaev ${ }^{1, *}$, Arthur Mazitov ${ }^{1}$ and Rustam Akhmetgaliev ${ }^{1}$ \\ ${ }^{1}$ LLC «RN-BashNIPIneft», Laboratory for research of anticorrosion technologies, Ufa, Russia
}

\begin{abstract}
The main purpose of this work is to assess the relevance of the requirements for the quantitative content of water-soluble salts on the metal surface before applying an anti-corrosion coating. To do this, a series of tests are carried out by coating the metal surface of samples with different salt content, followed by aging in distilled water at high temperature and pressure. After exposure, the defects will be analyzed. The results obtained will help to choose the maximum allowable amount of soluble salts on the metal surface, because the internal coating of field pipelines is practically beyond repair, and to fix this value in the regulatory document.
\end{abstract}

\section{Introduction}

Osmotic swelling is one of the most common causes of premature failure of anticorrosive coatings in contact with liquid media at high pressures and temperature. This is especially true for the oil industry, because still, protective anti-corrosion coating is one of the most popular methods of passive protection of the inner surface of oilfield pipelines and tanks. The source [1] has already said that this problem has been studied for many years by many experts in the field of research of the properties of anticorrosive coatings. The mechanism of this phenomenon has been studied and is well known, it lies in the fact that if the metal surface before applying the protective coating are water-soluble salts, and the protected object in contact with the liquid medium, the osmosis reaction is triggered. Accordingly, this leads to a complete loss of adhesive properties and sub-film corrosion of the metal [1].

In this work, the main attention is paid to the preparation of the inner surface of steel pipes and pipe products. The fact that the repair of the internal coating of these elements of the underground pipeline, is practically impossible, leads to the fact that it is necessary to revise the old rules and requirements for the cleanliness of the surface in the direction of their tightening.

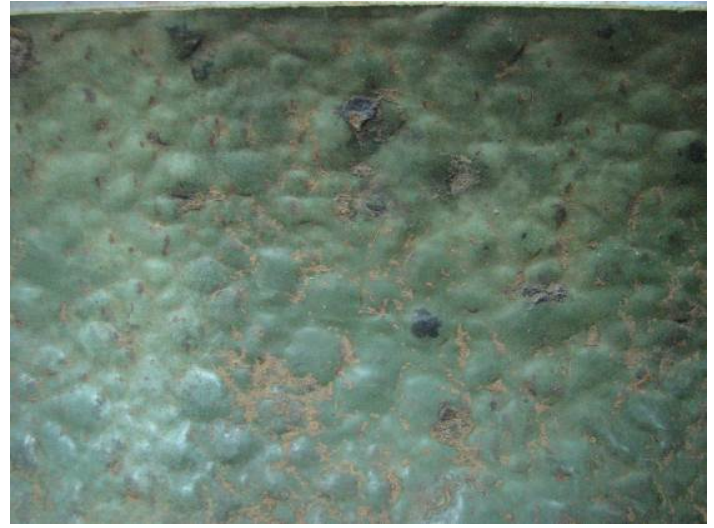

Fig. 1. Osmotic blistering of the internal anticorrosion coating of the pipeline.

\subsection{Control of soluble salts content on the surface of the metal}

One of the methods of protection against osmotic defects is the strict control of the preparation of the metal surface before applying paint coating. Of course, in the case of painting in ideal factory conditions to achieve the maximum permissible value of the salt content on the surface of the metal is no longer a problem. Control of this parameter is mainly carried out according to ISO $8502-2$. It should not be forgotten that the speed and intensity of the formation of defects in the form of osmotic blistering is affected not only by the salt content on the metal surface, but also by various operating

\footnotetext{
* Corresponding author: AtdaevAA@, bashneft.ru
} 
conditions - the pressure and temperature and the composition of the transported and stored medium.

\subsection{The norms for permissible content of soluble salts on the painted metal surface}

However, even excluding the human factor (noncompliance with the technological process of coating and not carrying out incoming inspection of products), defects of this kind, leading to premature failure of technological tanks and pipelines, are common. Perhaps the reason for this lies not in sufficient requirements for cleaning the metal from salts or in difficult operating conditions. governing the maximum permissible salt content on the metal surface before painting technological systems for the oil industry. At different producers of pipes and pipe products, with an internal anticorrosive paint and varnish coating, in technical documentation on finished products, the norm for the content of salts varies several times or is absent at all. But at the same time, the products of these manufacturers are intended for use in the same conditions.

The source [1] says that the analysis of studies in this direction revealed the range of the maximum permissible salt content from $20 \mathrm{mg} / \mathrm{m}^{2}$ to $100 \mathrm{mg} / \mathrm{m}^{2}$. This range of values is due to the dependence on the operating conditions of the protected structures for which the coatings were tested. If we turn to [2], it can be seen that in the aggregate, the results of studies are more suitable for structures in an industrial atmosphere with high humidity, and objects having constant or temporary contact with fresh and sea waters at relatively low temperatures and pressures.

All these requirements mainly relate to the use of protective coatings mainly on structures potentially suitable for repainting or partial repair of the coating.

But you can not just take and unreasonably increase or create norms that are not confirmed by the experimental base.

\section{Research on finding the maximum permissible content of water-soluble salts on the metal surface}

\subsection{The essence of the study}

Since the topic is quite serious, this type of research requires careful preparation and consideration of many factors that can affect the accuracy of the results. But, the process of preparation for the experiments, their conduct and analysis of the results will take a long time.

The essence of the test is to apply corrosion-resistant coating on steel samples with different salt content on the metal surface $\left(\mathrm{mg} / \mathrm{m}^{2}\right)$, as well as testing these plates in distilled water at high temperature, atmospheric and high pressure.

For the experiment, samples of paints intended to protect the internal surfaces of tanks, pipes and pipe products from corrosion provided by various manufacturers will be selected. For each coating there were 15 samples, 5 variants of the concentration of soluble salts on the surface of the metal at the same time for each concentration of 3 samples.

\subsection{The algorithm of the experiment}

\subsubsection{Test sample requirements}

Geometric dimensions of samples $150 \mathrm{~mm} \times 100 \mathrm{~mm} \times 3-5$ $\mathrm{mm}$, degree of purification $\mathrm{Sa} 2^{1 / 2}$ according to ISO $8501-1$, surface roughness of RZ from 40 to 80 microns according to GOST standard 2789-73. Samples should be cleaned of dust and degreased. Preparation of the sample consists in applying a solution of sodium salt of different concentrations on their surface with subsequent drying and determination of the obtained values of the salt content on the metal surface in $\mathrm{mg} / \mathrm{m}^{2}$ using the device Elcometer 130 model $\mathrm{T}$. The samples are placed either in a thermostat with distilled water at a temperature of $90 \pm 3 \mathrm{C}$ (or at a temperature recommended by the manufacturer of coatings), or in an autoclave with distilled water at a temperature of $80 \pm 3 \mathrm{C}$ (or at a temperature recommended by the manufacturer of coatings) and at a pressure of $10.0 \pm 0.5 \mathrm{MPa}$.

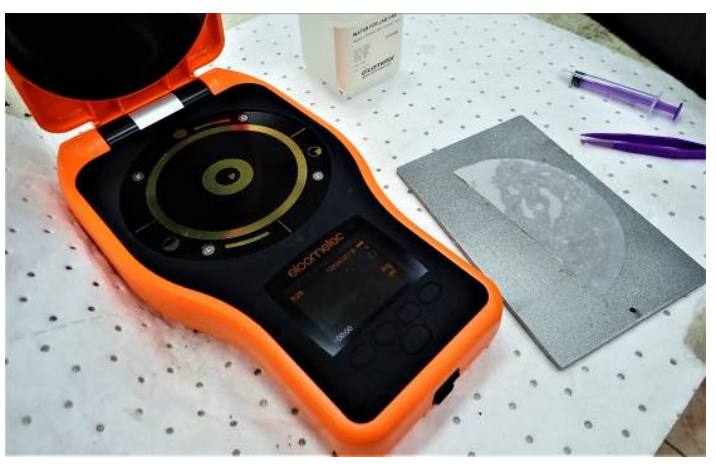

Fig. 2. Device for determination of water-soluble salts Elcometer 130 model T.

\subsubsection{The duration of the experiment}

In the case of the experiment in the thermostat duration is 1000 hours. In the case of an experiment in an autoclave, the duration of the experiment is 240 hours.

\subsubsection{Control of coated samples}

In case of exposure of samples in the thermostat:

1) control of changes in appearance is carried out visually every day;

2) detected changes in appearance are recorded in the test $\log$ and, if possible, should be photo-recorded;

3 ) if on the surface of one or more samples there are osmotic bubbles visible to the naked eye, the samples should be removed from the thermostat and recorded in the log the amount of salt content on the metal surface of the sample or samples and the time of their removal;

4) if bubbles were formed only on one sample of three (with one salt content), it is necessary to fix the time 
between the removal of the first and second sample to check repeatability.

In case of exposure of samples in the autoclave: 1) control of changes in appearance is carried out only on the day of the end of the test;

2) detected changes in appearance are recorded in the test log and, if possible, should be photo-recorded;

3 ) if osmotic bubbles appear on the surface of one or more samples that are visible to the naked eye, the salt content of the metal surface of the sample or samples should be recorded in the test log.

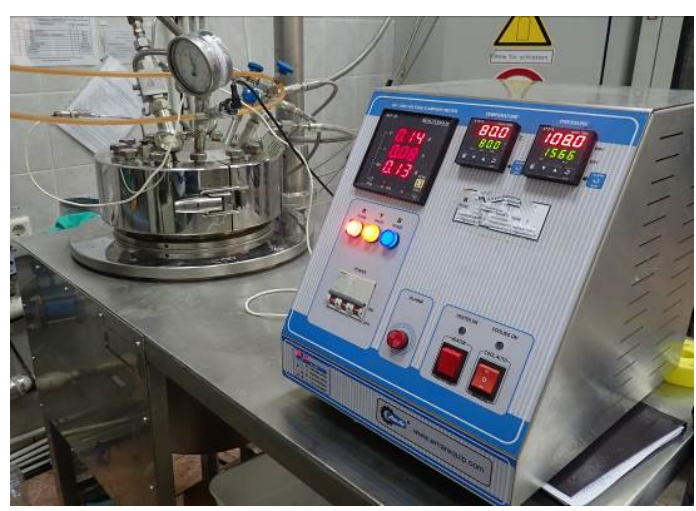

Fig. 3. Autoclave for the testing of anticorrosive coatings AMAR EQ.

\subsubsection{Results}

The results of the experiment will be evaluated according to ISO 4628-2:2003 standard designed to determine the degree of bubble formation and the degree of bloating.

In addition, metallographic sections from the zone of formation of osmotic bubbles will be made to study the nature of the destruction of the adhesive bonds of the metal with the coating and the presence of sub-film corrosion using an optical microscope.

\section{Discussion and conclusion}

According to the results of several cycles of the above experiments, it is desirable to obtain the maximum permissible value of soluble salts on the metal surface before applying the paint coating, which ensures an acceptable service life of pipes and pipe products.

In the future, we would like to be able to develop and apply methods that evaluate the effect of different content of soluble salts on the metal surface on the durability of the coating, as well as the comparative characteristics of the resistance of coatings to osmotic blistering.

\section{References}

1. D. de la Fuente, B. Chico, M. Morcillo, Port. Electrochim. Acta, 24, 191-206 (2006)

2. J.E.O. Mayne, J. Appl. Chem. 9, 673-680 (1959)

3. E.L. Koehler, Corrosion 33 (6), 209 (1977)
4. M. Morcillo, Prog. Org. Coat. 36, 137-147 (1999)

5. ISO/TR 15235:2001(E)

6. ISO 4628-2:2003(R) 